TRANSACTIONS OF THE

AMERICAN MATHEMATICAL SOCIETY

Volume 364, Number 10, October 2012, Pages 5265-5283

S 0002-9947(2012)05473-0

Article electronically published on May 29, 2012

\title{
HARMONIC MAP HEAT FLOW WITH ROUGH BOUNDARY DATA
}

\author{
LU WANG
}

\begin{abstract}
Let $B_{1}$ be the unit open disk in $\mathbb{R}^{2}$ and $M$ a closed Riemannian manifold. In this note, we first prove the uniqueness for weak solutions of the harmonic map heat flow in $H^{1}\left([0, T] \times B_{1}, M\right)$ whose energy is non-increasing in time, given initial data $u_{0} \in H^{1}\left(B_{1}, M\right)$ and boundary data $\gamma=\left.u_{0}\right|_{\partial B_{1}}$. Previously, this uniqueness result was obtained by Rivière (when $M$ is the round sphere and the energy of initial data is small) and Freire (when $M$ is an arbitrary closed Riemannian manifold), given that $u_{0} \in H^{1}\left(B_{1}, M\right)$ and $\gamma=\left.u_{0}\right|_{\partial B_{1}} \in H^{3 / 2}\left(\partial B_{1}\right)$. The point of our uniqueness result is that no boundary regularity assumption is needed. Second, we prove the exponential convergence of the harmonic map heat flow, assuming that energy is small at all times.
\end{abstract}

\section{INTRODUCTION}

Let $B_{1}$ be the unit open disk in $\mathbb{R}^{2}$ and $M$ a closed Riemannian manifold. Suppose that $u \in H^{1}\left([0, T] \times B_{1}, M\right)$ is the weak solution of the initial-boundary value problem for the harmonic map heat flow, given initial data $u_{0} \in H^{1}\left(B_{1}, M\right)$ and boundary data $\gamma=\left.u_{0}\right|_{\partial B_{1}} 11$ In this note, we study the uniqueness and the rate of convergence of the weak solution $u$.

Under the additional assumption that $\gamma \in H^{3 / 2}\left(\partial B_{1}, M\right)$, the initial-boundary value problem for the harmonic map heat flow has been investigated intensively by several mathematicians, such as Chang [2], Rivière [14] and Freire [7, [6] and [8]. Define

(1.1) $V^{T}=H^{1}\left([0, T] \times B_{1}, M\right) \cap L^{\infty}\left([0, T], H^{1}\left(B_{1}, M\right)\right) \cap L^{2}\left([0, T], H^{2}\left(B_{1}, M\right)\right)$.

Space $V^{T}$ plays a crucial role in the papers referred to above. However, without $\gamma \in H^{3 / 2}\left(\partial B_{1}\right)$, we are not able to show that $u$ with non-increasing energy is in $V^{T^{\prime}}$ for some $T^{\prime}>0$, as Freire did in 6]. Because, otherwise, it would imply that $\gamma \in H^{3 / 2}\left(\partial B_{1}, M\right)$ by the Sobolev trace theorem. But the image of trace operator on $H^{1}\left(B_{1}\right)$ is exactly $H^{1 / 2}\left(\partial B_{1}\right)$, and $H^{3 / 2}\left(\partial B_{1}\right)$ is a proper subset of $H^{1 / 2}\left(\partial B_{1}\right)$.

To get around this, we make use of the interior gradient estimate for $u$ and Hardy's inequality. The main difficulty is to deal with the $L^{2}$ inner product of $|\nabla u|^{2}$ and $h^{2}$ for $\forall h \in H_{0}^{1}\left(B_{1}\right)$, which arises from the non-linear term in the harmonic map heat flow equation. First, using Hélein's existence result of the Coulomb frame, we derive the interior gradient estimate for $u$ with small energy. Second, assuming the energy is non-increasing in time, we conclude that $u(t, \cdot) \longrightarrow u_{0}$ in the $H^{1}\left(B_{1}\right)$ topology, and thus we can uniformly bound the energy on small disks

Received by the editors July 22, 2010 and, in revised form, September 11, 2010.

2010 Mathematics Subject Classification. Primary 58E20; Secondary 35K55.

${ }^{1}$ The restriction here is the trace operator acting on $u_{0}$.

C) 2012 American Mathematical Society
Reverts to public domain 28 years from publication 
for short time. Thus, applying the gradient estimate for $u$ restricted on small disks, we bound $|\nabla u|^{2}(t, x)$ by $t^{-1}$ and $(1-|x|)^{-2}$ for short time. Finally, by Hardy's inequality, we bound the $L^{2}$ inner product of $|\nabla u|^{2}$ and $h^{2}$ for small time $t>0$.

In Section 5, we prove the existence and uniqueness theorem for weak solutions of the harmonic map heat flow in $\bigcap_{T>0} H^{1}\left([0, T] \times B_{1}, M\right)$ whose energy is nonincreasing in time, given small energy initial data $u_{0} \in H^{1} \cap C^{0}\left(\bar{B}_{1}\right)$ and boundary data $\gamma=\left.u_{0}\right|_{\partial B_{1}}$. By the Sobolev trace theorem, such weak solutions are not in $V^{T}$ in general.

Throughout, we use the subscripts $t, x_{1}, x_{2}$ and $r$ to denote taking derivatives with respect to $t, x_{1}, x_{2}$ and $r ; \nabla \cdot$ and $\nabla^{2}$. denote the gradient and the Hessian operator, respectively; "sup" in this note is "esssup" in the usual literature; constants in proofs are not preserved when crossing lemmas, propositions, and theorems.

By the Nash embedding theorem, $M$ can be isometrically embedded in some Euclidean space $\left(\mathbb{R}^{N},\langle\rangle,\right)$. Given $w \in H^{1}\left(B_{1}, M\right)$, we define the energy functional $E(w)=\frac{1}{2} \int_{B_{1}}|\nabla w|^{2}$. The harmonic map heat flow is the negative $L^{2}$ gradient flow of the energy functional. Thus, given $u_{0} \in H^{1}\left(B_{1}, M\right)$ and $\gamma=\left.u_{0}\right|_{\partial B_{1}}$, $u \in H^{1}\left([0, T] \times B_{1}, M\right) \sqrt{2}$ is the weak solution of the initial-boundary value problem for the harmonic map heat flow, if

$$
\begin{cases}u_{t}-\Delta u=-A_{u}(\nabla u, \nabla u) & \text { on } \quad(0, T) \times B_{1} \\ u(t, x)=\gamma(x) & \text { for } t \geq 0, x \in \partial B_{1}, \\ \lim _{t \rightarrow 0+} u(t, \cdot)=u_{0} & \text { in } L^{2}\left(B_{1}, M\right) \text { topology }\end{cases}
$$

where $A$ is the second fundamental form of $M$ in $\mathbb{R}^{N}$ at the point $u$. We recall that

$$
A_{u}(\nabla u, \nabla u) \text { is perpendicular to } M \text { at } u \text {. }
$$

We define the weak solution $u \in H^{1}\left([0, T] \times B_{1}, M\right)$ of the first equation in (1.2) by

$$
\int_{0}^{T} \int_{B_{1}}\left\langle u_{t}, \xi\right\rangle+\langle\nabla u, \nabla \xi\rangle+\left\langle A_{u}(\nabla u, \nabla u), \xi\right\rangle d x d t=0,
$$

for $\forall \xi \in C_{c}^{\infty}\left((0, T) \times B_{1}, \mathbb{R}^{N}\right)$. In [17], it is shown that this definition is equivalent to that, for a.e. $t \in[0, T]$,

$$
\int_{\{t\} \times B_{1}}\left\langle u_{t}, \zeta\right\rangle+\langle\nabla u, \nabla \zeta\rangle+\left\langle A_{u}(\nabla u, \nabla u), \zeta\right\rangle d x=0, \quad \forall \zeta \in H_{0}^{1} \cap L^{\infty}\left(B_{1}, \mathbb{R}^{N}\right) .
$$

Note that the definition given by equation (1.5) allows us to freeze the time and is more convenient for our proofs of Theorems 1.1 and 1.3

The main results of this note are twofold. First, we show the uniqueness for weak solutions of (1.2) whose energy is non-increasing.

Theorem 1.1. If $u$ and $v$ are weak solutions of (1.2) in $H^{1}\left([0, T] \times B_{1}, M\right)$ satisfying $E\left(u\left(t_{2}, \cdot\right)\right) \leq E\left(u\left(t_{1}, \cdot\right)\right), E\left(v\left(t_{2}, \cdot\right)\right) \leq E\left(v\left(t_{1}, \cdot\right)\right)$ for $t_{1} \leq t_{2}$, and having the same initial data $u_{0} \in H^{1}\left(B_{1}, M\right)$ and boundary value $\gamma=\left.u_{0}\right|_{\partial B_{1}}$, then $u=v$ on $[0, T] \times B_{1}$.

\footnotetext{
${ }^{2} \mathrm{By}$ Theorem 3 on page 287 of $\left[5\right.$, if $w \in H^{1}\left([0, T] \times B_{1}\right)$, then there exists $\tilde{w} \in$ $C^{0}\left([0, T], L^{2}\left(B_{1}\right)\right)$ and $\tilde{w}(t, \cdot)=w(t, \cdot)$ for a.e. $t$. Thus, in this note, we always choose $\tilde{w}$ representing $w$ in $H^{1}\left([0, T] \times B_{1}\right)$. In other words, we always assume that functions in $H^{1}\left([0, T] \times B_{1}\right)$ are actually also in $C^{0}\left([0, T], L^{2}\left(B_{1}\right)\right)$.
} 
Remark 1.2. In [1, Bertsch, Dal Passo and van der Hout proved that there exist initial data $u_{0} \in H^{1}\left(B_{1}, S^{2}\right)$ and boundary data $\gamma=\left.u_{0}\right|_{\partial B_{1}}$ such that (1.2) has infinitely many weak solutions which do not satisfy the non-increasing energy condition. Thus, Theorem 1.1 appears to be the optimal uniqueness statement for weak solutions of the harmonic map heat flow with time independent boundary data.

Second, we study the rate of convergence of small energy weak solutions of (1.2), and we conclude that

Theorem 1.3. There exists $\epsilon_{0}>0$, depending only on $M$, so that if $u$ is the weak solution of (1.2) in $\bigcap_{T>0} H^{1}\left([0, T] \times B_{1}, M\right)$ satisfying that $E(u(t, \cdot))<\epsilon_{0}$ for a.e. $t$, then there exist $T_{0}>0, \alpha_{0}>0$ and $C_{0}>0$ such that, for a.e $t>T_{0}$,

$$
\left\|u(t, \cdot)-u_{\infty}\right\|_{H^{1}} \leq C_{0} e^{-\alpha_{0} t}
$$

where $u_{\infty}$ is some harmonic map from $B_{1}$ to $M$ with the same boundary value $\gamma$.

Remark 1.4. It follows from Corollary 3.3 in [3] that $u_{\infty}$ is the unique harmonic map in the class of

$$
\left\{w \in H^{1}\left(B_{1}, M\right)|w|_{\partial B_{1}}=\gamma \text { and } E(w) \leq E_{1}\right\},
$$

where $E_{1}>0$ is a constant depending only on $M$.

Remark 1.5. Although Theorems 1.1 and 1.3 are stated for the unit open disk in this note, the proofs could be modified to apply to any bounded open set in $\mathbb{R}^{2}$ and even general two dimensional Riemannian manifolds.

\section{INTERIOR GRADIENT ESTIMATE FOR THE HARMONIC MAP HEAT FLOW}

In this section, we derive the interior gradient estimate for small energy solutions of the harmonic map heat flow. This is one of the key ingredients in the proofs of Theorems 1.1 and 1.3. First, using Hélein's existence result of the Coulomb frame, we show that $u(t, \cdot) \in H^{2}\left(B_{1 / 2}, M\right)$ for a.e. $t$. Next, we follow Struwe's method in [15] to conclude that $u \in L^{2}\left([0, \bar{T}], H^{2}\left(B_{1 / 4}, M\right)\right)$ for $1<\bar{T}<2$ and obtain the gradient estimate for $u$ at $(1,0,0)$.

The following elementary geometric fact is obtained in 4 and will be used frequently in this note. For self-containedness, we include the proof in Appendix A.

Lemma 2.1 (Lemma A.1 in 4]). There exists $C>0$, depending on $M$, so that if $x, y \in M$, then $\left|(x-y)^{\perp}\right| \leq C|x-y|^{2}$, where $(x-y)^{\perp}$ is the normal component to $M$ at $y$.

First, we derive the local integral bounds for $\left|\nabla^{2} u\right|$ and $|\nabla u|$ :

Lemma 2.2. Suppose that $1<\bar{T}<2$ and $u \in H^{1}\left([0, \bar{T}] \times B_{1}, M\right)$ satisfies

$$
u_{t}-\Delta u=-A_{u}(\nabla u, \nabla u)
$$

on $(0, \bar{T}) \times B_{1}$. Then there exists $\epsilon>0$, depending only on $M$, so that if $E(u(t, \cdot)) \leq$ $\epsilon$ for a.e. $t \in[0, \bar{T}]$, then

$$
\begin{aligned}
& \int_{0}^{\bar{T}} \int_{B_{1 / 4}}\left|\nabla^{2} u\right|^{2} d x d t \leq 10^{5} \sup _{0 \leq t \leq \bar{T}} E(u(t, \cdot)), \\
& \int_{0}^{\bar{T}} \int_{B_{1 / 4}}|\nabla u|^{4} d x d t \leq 10^{8} \sup _{0 \leq t \leq \bar{T}} E(u(t, \cdot))^{2} .
\end{aligned}
$$


Proof. First, note that $\left|u_{t}\right| \in L^{2}\left(B_{1}\right)$ for a.e. $t$. Fix such $t$. Following the proof of Theorem 4.1.1 in [11, there exists $\delta_{1}>0$, depending only on $M$, so that if $E(u(t, \cdot)) \leq \delta_{1}$, then there is a finite energy harmonic section (i.e., "Coulomb frame" $) e(t)=\left(e_{1}(t), \ldots, e_{n}(t)\right)$ of the bundle of orthonormal frames for $u(t, \cdot)^{*}(T M)$, and one can construct $\beta(t) \in L^{\infty}\left(B_{1}, G L(n, \mathbb{C})\right)$ satisfying that $|\beta(t)| \leq \lambda_{1},\left|\beta(t)^{-1}\right| \leq \lambda_{1}$, and

$$
\partial_{\bar{z}}\left(\beta^{-1} \alpha(t)\right)=\frac{1}{4} \beta(t)^{-1} f,
$$

where $\lambda_{1}$ depends only on $M$ and the upper bound of the energy of $u(t, \cdot), z=$ $x_{1}+i x_{2}, \alpha=\left(\left\langle\partial_{z} u, e_{1}\right\rangle, \ldots,\left\langle\partial_{z} u, e_{n}\right\rangle\right)$ and $f=\left(\left\langle u_{t}, e_{1}\right\rangle, \ldots,\left\langle u_{t}, e_{n}\right\rangle\right)$. Thus, by the elliptic regularity for $\partial_{\bar{z}}$ operator (see the theorem on page 80 of [10]), $\beta^{-1} \alpha(t) \in$ $H^{1}\left(B_{3 / 4}\right)$. It follows from the Sobolev embedding theorem (see Theorem 2 on page 265 of [5]) and $|\beta(t)| \leq \lambda_{1}$ that $\alpha(t) \in L^{p}\left(B_{3 / 4}\right)$ for $1<p<\infty$. In particular, $|\nabla u|(t, \cdot) \in L^{4}\left(B_{3 / 4}\right)$. Therefore, by Theorem 8.8 in [9], $u(t, \cdot) \in H^{2}\left(B_{1 / 2}\right)$.

Next, let $\phi$ be a smooth cut-off function, which is one in $B_{1 / 4}$, compactly supported in $B_{1 / 2}, 0 \leq \phi \leq 1$ and $|\nabla \phi| \leq 8$. Thus, by Lemma 6.7 in Chapter III of 16] and equation (2.1),

$$
\begin{aligned}
& \int_{\{t\} \times B_{1}}|\Delta u|^{2} \phi^{2} d x \leq \int_{\{t\} \times B_{1}}\left|u_{t}\right|^{2} \phi^{2} d x+\sup _{M}|A|^{2} \int_{\{t\} \times B_{1}}|\nabla u|^{4} \phi^{2} \\
\leq & \int_{\{t\} \times B_{1}}\left|u_{t}\right|^{2} \phi^{2} d x+\lambda_{2} E(u(t, \cdot))\left(\int_{\{t\} \times B_{1}}\left|\nabla^{2} u\right|^{2} \phi^{2} d x+\int_{\{t\} \times B_{1}}|\nabla u|^{2} d x\right),
\end{aligned}
$$

where $\lambda_{2}=512 \sup _{M}|A|^{2}$. On the other hand, approximating $u(t, \cdot)$ by smooth functions in $H^{2}\left(B_{1 / 2}\right)$ and integration by parts, give

$$
\int_{\{t\} \times B_{1}}|\Delta u|^{2} \phi^{2} d x \geq \frac{1}{2} \int_{\{t\} \times B_{1}}\left|\nabla^{2} u\right|^{2} \phi^{2} d x-8 \int_{\{t\} \times B_{1}}|\nabla u|^{2}|\nabla \phi|^{2} d x .
$$

If $4 \lambda_{2} E(u(t, \cdot)) \leq 1$, then

$$
\int_{\{t\} \times B_{1}}\left|\nabla^{2} u\right|^{2} \phi^{2} d x \leq 32 \int_{\{t\} \times B_{1}}\left[\left|u_{t}\right|^{2} \phi^{2}+|\nabla u|^{2}\left(1+|\nabla \phi|^{2}\right)\right] d x .
$$

Thus, integrating over $[0, \bar{T}]$, we have

$$
\int_{0}^{\bar{T}} \int_{B_{1}}\left|\nabla^{2} u\right|^{2} \phi^{2} d x d t \leq 32 \int_{0}^{\bar{T}} \int_{B_{1}}\left[\left|u_{t}\right|^{2} \phi^{2}+|\nabla u|^{2}\left(1+|\nabla \phi|^{2}\right)\right] d x d t
$$

if $E(u(t, \cdot)) \leq \min \left\{\delta_{1}, \lambda_{2}^{-1} / 4\right\}$ for a.e. $t$. And it follows from the proof of Lemma 3.4 in [15] (replacing the test function $u$ by $u \phi^{2}$ ) that

$$
\int_{0}^{\bar{T}} \int_{B_{1}}\left|u_{t}\right|^{2} \phi^{2} d x d t \leq 1026 \sup _{0 \leq t \leq \bar{T}} E(u(t, \cdot)) .
$$

Therefore,

$$
\int_{0}^{\bar{T}} \int_{B_{1}}\left|\nabla^{2} u\right|^{2} \phi^{2} d x d t \leq 10^{5} \sup _{0 \leq t \leq \bar{T}} E(u(t, \cdot)),
$$


and it follows from Lemma 6.7 in Chapter III of [16] that

$$
\begin{aligned}
\int_{0}^{\bar{T}} \int_{B_{1}}|\nabla u|^{4} \phi^{4} d x d t & \leq 8 \sup _{0 \leq t \leq \bar{T}} E(u(t, \cdot)) \int_{0}^{\bar{T}} \int_{B_{1}}\left(|\nabla \phi|^{2}|\nabla u|^{2}+\left|\nabla^{2} u\right|^{2} \phi^{2}\right) d x d t \\
& \leq 10^{8} \sup _{0 \leq t \leq \bar{T}} E(u(t, \cdot))^{2}
\end{aligned}
$$

Now we are ready to prove the interior gradient estimate:

Lemma 2.3. Under the assumption of Lemma 2.2 , there exist $\epsilon_{1} \in(0, \epsilon]$ and $C_{1}>0$, depending only on $M$, so that if $E(u(t, \cdot)) \leq \epsilon_{1}$ for a.e. $t \in[0, \bar{T}]$, then

$$
|\nabla u|^{2}(1,0,0) \leq C_{1} \sup _{0 \leq t \leq \bar{T}} E(u(t, \cdot)) .
$$

Proof. We will follow the suggestion in the remark after Lemma 3.10 in [15] to obtain the interior gradient estimate for $u$. Let $\phi$ be a smooth cut-off function, which is one in $B_{1 / 8}$, compactly supported in $B_{1 / 4}, 0 \leq \phi \leq 1$ and $|\nabla \phi| \leq 16$. Also, we define $D^{h} w(t, x)=(w(t+h, x)-w(t, x)) / h$ for $0<h<h_{0} \ll 1$, where $w$ takes value in $\mathbb{R}$ or $\mathbb{R}^{N}$. Thus, for $0<t_{1} \leq t_{2} \leq \bar{T}-h_{0}$, using equation (2.1) and integration by parts, we get

$$
\begin{aligned}
& \int_{t_{1}}^{t_{2}} \int_{B_{1}} \partial_{t}\left|D^{h} u\right|^{2} \phi^{2} d x d t+2 \int_{t_{1}}^{t_{2}} \int_{B_{1}}\left|\nabla D^{h} u\right|^{2} \phi^{2} d x d t \\
\leq & 4 \int_{t_{1}}^{t_{2}} \int_{B_{1}}\left|D^{h} u\right||\phi|\left|\nabla D^{h} u\right||\nabla \phi| d x d t+2 h^{-1} \int_{t_{1}}^{t_{2}} \int_{B_{1}}\left\langle A_{u}(\nabla u, \nabla u), D^{h} u\right\rangle \phi^{2} d x d t \\
& -2 h^{-1} \int_{t_{1}}^{t_{2}} \int_{B_{1}}\left\langle A_{u(t+h, x)}(\nabla u, \nabla u), D^{h} u\right\rangle \phi^{2} d x d t \\
\leq & 4 \int_{t_{1}}^{t_{2}} \int_{B_{1}}\left|D^{h} u\right||\phi|\left|\nabla D^{h} u\right||\nabla \phi| d x d t+\lambda_{1} \int_{t_{1}}^{t_{2}} \int_{B_{1}}\left|D^{h} u\right|^{2}|\nabla u|^{2}(t, x) \phi^{2} d x d t \\
& +\lambda_{1} \int_{t_{1}}^{t_{2}} \int_{B_{1}}\left|D^{h} u\right|^{2}|\nabla u|^{2}(t+h, x) \phi^{2} d x d t \\
\leq & \int_{t_{1}}^{t_{2}} \int_{B_{1}}^{t_{2}}\left|\nabla D^{h} u\right|^{2} \phi^{2} d x d t+4 \int_{t_{1}}^{t_{2}} \int_{B_{1}}\left|D^{h} u\right|^{2}|\nabla \phi|^{2} d x d t \\
& +\lambda_{1} \int_{t_{1}}^{t_{2}} \int_{B_{1}}\left|D^{h} u\right|^{2}\left(|\nabla u|^{2}(t, x)+|\nabla u|^{2}(t+h, x)\right) \phi^{2} d x d t,
\end{aligned}
$$

where $\lambda_{1}=C \sup _{M}|A|$, and we use (1.3) and Lemma 2.1] in the second inequality. Thus, absorbing the first term on the right-hand side in the left, gives

$$
\begin{aligned}
& \int_{\left\{t_{2}\right\} \times B_{1}}\left|D^{h} u\right|^{2} \phi^{2} d x+\int_{t_{1}}^{t_{2}} \int_{B_{1}}\left|\nabla D^{h} u\right|^{2} \phi^{2} d x d t \\
\leq & \int_{\left\{t_{1}\right\} \times B_{1}}\left|D^{h} u\right|^{2} \phi^{2} d x+4 \int_{t_{1}}^{t_{2}} \int_{B_{1}}\left|D^{h} u\right|^{2}|\nabla \phi|^{2} d x d t \\
& +\lambda_{1} \int_{t_{1}}^{t_{2}} \int_{B_{1}}\left|D^{h} u\right|^{2}\left(|\nabla u|^{2}(t, x)+|\nabla u|^{2}(t+h, x)\right) \phi^{2} d x d t .
\end{aligned}
$$


By Lemma 6.7 in Chapter III of [16, we get

$$
\begin{aligned}
& \int_{t_{1}}^{t_{2}} \int_{B_{1}}\left|D^{h} u\right|^{4} \phi^{4} d x d t \\
\leq & 8 \sup _{t_{1} \leq t \leq t_{2}} \int_{\{t\} \times B_{1}}\left|D^{h} u\right|^{2} \phi^{2} d x \cdot \int_{t_{1}}^{t_{2}} \int_{B_{1}}\left(\left|D^{h} u\right|^{2}|\nabla \phi|^{2}+\left|\nabla D^{h} u\right|^{2} \phi^{2}\right) d x d t .
\end{aligned}
$$

If $8 \lambda_{1} \sup _{0 \leq t \leq \bar{T}} E(u(t, \cdot))<10^{-4}$, then, by Lemma 2.2 and Hölder's inequality, we have

$$
\begin{aligned}
& \lambda_{1} \int_{t_{1}}^{t_{2}} \int_{B_{1}}\left|D^{h} u\right|^{2}\left(|\nabla u|^{2}(t, x)+|\nabla u|^{2}(t+h, x)\right) \phi^{2} d x d t \\
\leq & 2 \lambda_{1}\left(\int_{t_{1}}^{t_{2}} \int_{B_{1}}\left|D^{h} u\right|^{4} \phi^{4} d x d t\right)^{\frac{1}{2}}\left(\int_{t_{1}}^{t_{2}} \int_{B_{1 / 4}}\left(|\nabla u|^{4}(t, x)+|\nabla u|^{4}(t+h, x)\right) d x d t\right)^{\frac{1}{2}} \\
\leq & \left(\sup _{t_{1} \leq t \leq t_{2}} \int_{\{t\} \times B_{1}}\left|D^{h} u\right|^{2} \phi^{2} d x\right)^{\frac{1}{2}}\left(\int_{t_{1}}^{t_{2}} \int_{B_{1}}\left(\left|D^{h} u\right|^{2}|\nabla \phi|^{2}+\left|\nabla D^{h} u\right|^{2} \phi^{2}\right) d x d t\right)^{\frac{1}{2}} \\
\leq & \frac{1}{2} \sup _{t_{1} \leq t \leq t_{2}} \int_{\{t\} \times B_{1}}\left|D^{h} u\right|^{2} \phi^{2} d x+\frac{1}{2} \int_{t_{1}}^{t_{2}} \int_{B_{1}}\left(\left|D^{h} u\right|^{2}|\nabla \phi|^{2}+\left|\nabla D^{h} u\right|^{2} \phi^{2}\right) d x d t .
\end{aligned}
$$

Hence, (2.11) gives

$$
\begin{aligned}
& \int_{\left\{t_{2}\right\} \times B_{1}}\left|D^{h} u\right|^{2} \phi^{2} d x-\int_{\left\{t_{1}\right\} \times B_{1}}\left|D^{h} u\right|^{2} \phi^{2} d x \\
\leq & \frac{9}{2} \int_{t_{1}}^{t_{2}} \int_{B_{1}}\left|D^{h} u\right|^{2}|\nabla \phi|^{2} d x d t+\frac{1}{2} \sup _{t_{1} \leq t \leq t_{2}} \int_{\{t\} \times B_{1}}\left|D^{h} u\right|^{2} \phi^{2} d x .
\end{aligned}
$$

We conclude from (2.12) that

$$
\int_{\left\{t_{2}\right\} \times B_{1}}\left|D^{h} u\right|^{2} \phi^{2} d x \leq 2 \inf _{0 \leq t \leq t_{2}} \int_{\{t\} \times B_{1}}\left|D^{h} u\right|^{2} \phi^{2} d x+\lambda_{2} \int_{0}^{t_{2}} \int_{B_{1 / 4}}\left|D^{h} u\right|^{2} d x d t,
$$

where $\lambda_{2}>0$ is a universal constant. Therefore,

$$
\int_{\left\{t_{2}\right\} \times B_{1}}\left|D^{h} u\right|^{2} \phi^{2} d x \leq 2\left(t_{2}^{-1}+\lambda_{2}\right) \int_{0}^{t_{2}} \int_{B_{1 / 4}}\left|D^{h} u\right|^{2} d x d t .
$$

Note that $u$ is in $H^{1}\left([0, \bar{T}] \times B_{1}\right)$ and thus

$$
\int_{0}^{\bar{T}-h_{0}} \int_{B_{1}}\left|D^{h} u-u_{t}\right|^{2} d x d t \leq \int_{0}^{1} \int_{0}^{\bar{T}-h_{0}} \int_{B_{1}}\left|u_{t}(t+s h, x)-u_{t}(t, x)\right|^{2} d x d t d s .
$$

Since elements in $L^{2}$ are continuous in the mean,

$$
\lim _{h \longrightarrow 0} \int_{0}^{\bar{T}-h_{0}} \int_{B_{1}}\left|D^{h} u-u_{t}\right|^{2} d x d t=0 .
$$

Therefore, letting $h \longrightarrow 0$, by the arbitrariness of $h_{0},(2.13)$ and (2.8), we conclude that for a.e. $t$,

$$
\int_{\{t\} \times B_{1}}\left|u_{t}\right|^{2} \phi^{2} d x \leq 10^{4}\left(t^{-1}+\lambda_{2}\right) \sup _{0 \leq s \leq \bar{T}} E(u(s, \cdot)) .
$$


By the same argument used to derive (2.6), for $\frac{1}{2}<t<\bar{T}$,

$$
\int_{\{t\} \times B_{1}}\left|\nabla^{2} u\right|^{2} \phi^{2} d x \leq \lambda_{3} \sup _{0 \leq s \leq \bar{T}} E(u(s, \cdot))
$$

where $\lambda_{3}>0$ is a universal constant. Hence, by the Sobolev embedding theorem, for $1<p<\infty$,

$$
\int_{\frac{1}{2}}^{\bar{T}} \int_{B_{1 / 8}}|\nabla u|^{p} d x d t \leq \lambda_{4} \sup _{0 \leq t \leq \bar{T}} E(u(t, \cdot))^{\frac{p}{2}}
$$

where $\lambda_{4}$ depends only on $p$. Thus, by inserting cut-off functions and the theorem on page 72 of [10, $\left|u_{t}\right|$ and $\left|\nabla^{2} u\right|$ are in $L^{p}\left(\left[1 / 4, \bar{T}_{1}\right] \times B_{1 / 16}\right)$ for $1<\bar{T}_{1}<\bar{T}$. Furthermore, using the Bochner formula and the Gauss equation, one can derive the evolution equation for $g=|\nabla u|^{2}$ (see page 128 of [10]), that is,

$$
g_{t}-\Delta g=-2\left|\operatorname{Hess}_{u}\right|^{2}+2\left\langle A_{u}\left(u_{x_{1}}, u_{x_{1}}\right), A_{u}\left(u_{x_{2}}, u_{x_{2}}\right)\right\rangle-2\left|A_{u}\left(u_{x_{1}}, u_{x_{2}}\right)\right|^{2}
$$

Thus, $g \in W^{1, p}\left(\left[1 / 8, \bar{T}_{2}\right] \times B_{1 / 32}\right)$ and $\left|\nabla^{2} g\right| \in L^{p}\left(\left[1 / 8, \bar{T}_{2}\right] \times B_{1 / 32}\right)$ for $1<\bar{T}_{2}<\bar{T}_{1}$. Therefore, by the local maximum principle (see Theorem 7.36 in [12]),

$$
g(1,0,0)=|\nabla u|^{2}(1,0,0) \leq \lambda_{5} \sup _{0 \leq t \leq \bar{T}} E(u(t, \cdot)),
$$

where $\lambda_{5}>0$ depends only on $M$, assuming that $E(u(t, \cdot))<\epsilon_{1}$ for a.e. $t$ and $\epsilon_{1}=\min \left\{\epsilon, 10^{-4} \lambda_{1}^{-1} / 8\right\}$.

\section{Proof of Theorem 1.1}

In [6] and [8, Freire first constructed the optimal tangent frames for each fixed time and rewrote equation (2.1) under these frames. Next, he used the parabolic perturbation argument to show that any weak solution $u \in H^{1}\left([0, T] \times B_{1}, M\right)$ of (1.2), satisfying $E(u(t, \cdot)) \leq E\left(u_{0}\right)$ for a.e. $t \in[0, T]$, is in $V^{T^{\prime}}$ for some $T^{\prime} \in(0, T)$, given initial data $u_{0} \in H^{1}\left(B_{1}, M\right)$ and boundary data $\gamma=\left.u_{0}\right|_{\partial B_{1}} \in H^{3 / 2}\left(B_{1}, M\right)$; see Theorem 1.1 in [6]. Finally, combining with the results of Struwe in [15] and Chang in 2, he concluded the uniqueness for energy non-increasing weak solutions of (1.2) on $[0, T] \times B_{1}$ by iteration. However, without the assumption on boundary regularity, the proof by Freire does not apply to our case (as explained in the second paragraph of the Introduction). Instead, we make use of the interior gradient estimate and Hardy's inequality to show the uniqueness for energy non-increasing weak solutions of (1.2) in $H^{1}\left([0, T] \times B_{1}, M\right)$ with initial data $u_{0} \in H^{1}\left(B_{1}, M\right)$ and boundary data $\gamma=\left.u_{0}\right|_{\partial B_{1}}$.

We start with showing Hardy's inequality for the unit open disk. This turns out to be the other key ingredient. Such a Hardy's inequality also holds for general domains in $\mathbb{R}^{2}$; see [13].

Lemma 3.1. For $h \in H_{0}^{1}\left(B_{1}, \mathbb{R}\right)$,

$$
\int_{B_{1}} \frac{h^{2}}{\left(1-\sqrt{x_{1}^{2}+x_{2}^{2}}\right)^{2}} d x \leq 4 \int_{B_{1}}|\nabla h|^{2} d x .
$$


Proof. First, we prove the lemma for $h \in C_{c}^{\infty}\left(B_{1}, \mathbb{R}\right)$. Rewriting the left-hand side of inequality (3.1) in polar coordinates and using integration by parts, we get

$$
\begin{aligned}
& \int_{B_{1}} \frac{h^{2}}{\left(1-\sqrt{x_{1}^{2}+x_{2}^{2}}\right)^{2}} d x=\int_{0}^{1} \int_{0}^{2 \pi} \frac{h^{2} r}{(1-r)^{2}} d \theta d r \\
= & -\int_{0}^{1} \int_{0}^{2 \pi} \frac{h^{2}}{1-r} d \theta d r-\int_{0}^{1} \int_{0}^{2 \pi} \frac{2 h h_{r} r}{1-r} d \theta d r \\
\leq & 2\left(\int_{0}^{1} \int_{0}^{2 \pi} \frac{h^{2} r}{(1-r)^{2}} d \theta d r\right)^{\frac{1}{2}}\left(\int_{0}^{1} \int_{0}^{2 \pi} h_{r}^{2} r d \theta d r\right)^{\frac{1}{2}} \\
\leq & 2\left(\int_{B_{1}} \frac{h^{2}}{\left(1-\sqrt{x_{1}^{2}+x_{2}^{2}}\right)^{2}} d x\right)^{\frac{1}{2}}\left(\int_{B_{1}}|\nabla h|^{2} d x\right)^{\frac{1}{2}} .
\end{aligned}
$$

Thus, inequality (3.1) follows by absorbing the second term of the product on the right-hand side in the left.

Since $C_{c}^{\infty}\left(B_{1}\right)$ is dense in $H_{0}^{1}\left(B_{1}\right)$, there exists a sequence of $h_{n} \in C_{c}^{\infty}\left(B_{1}, \mathbb{R}\right)$ such that $h_{n} \longrightarrow h$ in $H^{1}\left(B_{1}\right)$ topology and $h_{n} \longrightarrow h$ a.e. in $B_{1}$. By Fatou's lemma (see Theorem 3 on page 648 of [5]),

$$
\begin{aligned}
\int_{B_{1}} \frac{h^{2}}{\left(1-\sqrt{x_{1}^{2}+x_{2}^{2}}\right)^{2}} d x & \leq \liminf _{n \longrightarrow \infty} \int_{B_{1}} \frac{h_{n}^{2}}{\left(1-\sqrt{x_{1}^{2}+x_{2}^{2}}\right)^{2}} d x \\
& \leq \liminf _{n \longrightarrow \infty} 4 \int_{B_{1}}\left|\nabla h_{n}\right|^{2} d x=4 \int_{B_{1}}|\nabla h|^{2} d x .
\end{aligned}
$$

Next, to avoid repeating computation in Section 5 , we will prove a general stability lemma below, i.e., Lemma 3.2. Suppose that $u$ and $v$ are weak solutions of (1.2) in $H^{1}\left([0, T] \times B_{1}, M\right)$ satisfying that the energy is non-increasing and with initial data $u_{0}$ and $v_{0}$, respectively. For this moment, $u_{0}$ may not be equal to $v_{0}$. And let $\epsilon_{2}=\min \left\{\epsilon_{1}, C^{-1} C_{1}^{-1} \sup _{M}|A| / 32\right\}$.

The key to the proof of Lemma 3.2 is to bound the $L^{2}$ inner products $\left\langle|\nabla u|^{2}, h^{2}\right\rangle_{L^{2}}$ and $\left\langle|\nabla v|^{2}, h^{2}\right\rangle_{L^{2}}$ on $B_{1}$, for $\forall h \in H_{0}^{1}\left(B_{1}\right)$. Such integrals arise from the non-linear terms $A_{u}(\nabla u, \nabla u)$ and $A_{v}(\nabla v, \nabla v)$ in equation (2.1). First, by the energy nonincreasing assumption and Lemma 2.3, we can bound $|\nabla u|$ and $|\nabla v|$ for $x_{0} \in B_{1}$ and small time $t_{0}>0$. Namely, since the energy of $u(t, \cdot)$ is non-increasing in time, $u(t, \cdot) \longrightarrow u_{0}$ weakly in $H^{1}\left(B_{1}\right)$ and strongly in $L^{2}\left(B_{1}\right)$, as $t \longrightarrow 0$. Then,

$$
\begin{aligned}
& \lim _{t \rightarrow 0} \int_{B_{1}}\left|\nabla u(t, x)-\nabla u_{0}\right|^{2} d x \\
= & \lim _{t \rightarrow 0} \int_{B_{1}}|\nabla u(t, x)|^{2} d x-\int_{B_{1}} 2\left\langle\nabla u(t, x), \nabla u_{0}\right\rangle d x+\int_{B_{1}}\left|\nabla u_{0}\right|^{2} d x \\
\leq & 0 .
\end{aligned}
$$

Thus, $u(t, \cdot) \longrightarrow u_{0}$ strongly in $H^{1}\left(B_{1}\right)$, and by the same argument, $v(t, \cdot) \longrightarrow v_{0}$ strongly in $H^{1}\left(B_{1}\right)$, as $t \longrightarrow 0$. Hence, by the absolute continuity of integration, there exist $R_{0}>0$ and $T^{\prime} \in\left(0, \min \left\{R_{0}^{2}, T\right\}\right]$ so that for $x_{0} \in B_{1}$ and $t \in\left[0, T^{\prime}\right]$,

$$
\frac{1}{2} \int_{\{t\} \times\left(B_{R_{0}}\left(x_{0}\right) \cap B_{1}\right)}|\nabla u|^{2} d x<\epsilon_{2} \quad \text { and } \quad \frac{1}{2} \int_{\{t\} \times\left(B_{R_{0}}\left(x_{0}\right) \cap B_{1}\right)}|\nabla v|^{2} d x<\epsilon_{2} .
$$

Note that equation (2.1) is invariant under the transformation $(t, x) \longrightarrow\left(\lambda^{2} t, \lambda x\right)$ for $\lambda>0$, and the energy is invariant under conformal transformations of domains 
in $\mathbb{R}^{2}$. Fix $\left(t_{0}, x_{0}\right) \in\left(0, T^{\prime}\right) \times B_{1}$. Let $\lambda=\min \left\{\sqrt{t_{0}}, 1-\left|x_{0}\right|\right\}$. Define $u_{\lambda}(s, y)=$ $u\left(\lambda^{2} s, x_{0}+\lambda y\right)$ and $v_{\lambda}(s, y)=v\left(\lambda^{2} s, x_{0}+\lambda y\right)$. Thus, $u_{\lambda}$ and $v_{\lambda}$ satisfy equation (2.1) on $\left(0, \lambda^{-2} T^{\prime}\right) \times B_{1}$, and for $s \in\left[0, \lambda^{-2} T^{\prime}\right], E\left(u_{\lambda}(s, \cdot)\right)<\epsilon_{2}$ and $E\left(v_{\lambda}(s, \cdot)\right)<\epsilon_{2}$. Hence, by Lemma 2.3. for $\left(t_{0}, x_{0}\right) \in\left(0, T^{\prime}\right) \times B_{1}$,

$$
\left|\nabla u_{\lambda}\right|^{2}\left(\lambda^{-2} t_{0}, 0,0\right) \leq C_{1} \epsilon_{2} \quad \text { and } \quad\left|\nabla v_{\lambda}\right|^{2}\left(\lambda^{-2} t_{0}, 0,0\right) \leq C_{1} \epsilon_{2} .
$$

Therefore,

$$
\begin{aligned}
& |\nabla u|^{2}\left(t_{0}, x_{0}\right) \leq C_{1}\left[t_{0}^{-1}+\left(1-\left|x_{0}\right|\right)^{-2}\right] \epsilon_{2}, \\
& |\nabla v|^{2}\left(t_{0}, x_{0}\right) \leq C_{1}\left[t_{0}^{-1}+\left(1-\left|x_{0}\right|\right)^{-2}\right] \epsilon_{2} .
\end{aligned}
$$

Then, combining inequalities (3.4) and (3.5) with Lemma 3.1 we can bound the $L^{2}$ inner products $\left\langle|\nabla u|^{2}, h^{2}\right\rangle_{L^{2}}$ and $\left\langle|\nabla v|^{2}, h^{2}\right\rangle_{L^{2}}$ on $B_{1}$, for $\forall h \in H_{0}^{1}\left(B_{1}\right)$ and $t \in\left(0, T^{\prime}\right)$.

Lemma 3.2. There exists $C_{2}>0$, depending only on $M$, so that

$$
\int_{0}^{T^{\prime}} \int_{B_{1}}|\nabla u-\nabla v|^{2} t^{-\frac{1}{2}} d x d t+\frac{1}{2 \sqrt{T^{\prime}}} \int_{\left\{T^{\prime}\right\} \times B_{1}}|u-v|^{2} d x \leq N
$$

where

$$
\begin{aligned}
N= & \left(\frac{1}{\sqrt{T^{\prime}}}+2 \sqrt{T^{\prime}}\right) \int_{B_{1}}\left(\left|w_{0}\right|^{2}+\left|\nabla w_{0}\right|^{2}\right) d x \\
& +8 \sqrt{2 T^{\prime}\left(E\left(u_{0}\right)+E\left(v_{0}\right)\right)}\left(\int_{B_{1}}\left|\nabla w_{0}\right|^{2} d x\right)^{\frac{1}{2}} \\
& +4 C_{2} \int_{0}^{T^{\prime}} \int_{B_{1}}\left(|\nabla u|^{2}+|\nabla v|^{2}\right)\left(\left|w_{0}\right|^{2}+\left|w_{0}\right|\right) t^{-\frac{1}{2}} d x d t .
\end{aligned}
$$

Proof. Define $w=u-v$. It is clear that

$$
\begin{aligned}
& \int_{0}^{T^{\prime}} \int_{B_{1}}|\nabla w|^{2} t^{-\frac{1}{2}} d x d t \\
= & \int_{0}^{T^{\prime}} \int_{B_{1}}\langle\nabla u, \nabla w\rangle t^{-\frac{1}{2}} d x d t-\int_{0}^{T^{\prime}} \int_{B_{1}}\langle\nabla v, \nabla w\rangle t^{-\frac{1}{2}} d x d t .
\end{aligned}
$$

We will estimate the first term of (3.8) and the second term can be estimated similarly. First, by footnote $2, w-w_{0} \in C^{0}\left([0, T], L^{2}\left(B_{1}\right)\right)$ and the map $t \longrightarrow$ $\left\|w(t, \cdot)-w_{0}\right\|_{L^{2}\left(B_{1}\right)}^{2}$ is absolutely continuous, with

$$
\begin{aligned}
\frac{d}{d t}\left\|w(t, \cdot)-w_{0}\right\|_{L^{2}\left(B_{1}\right)}^{2} & =2 \int_{\{t\} \times B_{1}}\left\langle w_{t}, w-w_{0}\right\rangle d x \\
& \leq 2\left(\int_{\{t\} \times B_{1}}\left|w_{t}\right|^{2} d x\right)^{\frac{1}{2}}\left(\int_{\{t\} \times B_{1}}\left|w-w_{0}\right|^{2} d x\right)^{\frac{1}{2}}
\end{aligned}
$$


for a.e. $t \in[0, T]$. Thus, for $\forall t_{0} \geq 0$, integrating over $\left[0, t_{0}\right]$ and by Hölder's inequality, we conclude that

$$
\begin{aligned}
\left\|w\left(t_{0}, \cdot\right)-w_{0}\right\|_{L^{2}\left(B_{1}\right)} & \leq \int_{0}^{t_{0}}\left(\int_{\{t\} \times B_{1}}\left|w_{t}\right|^{2} d x\right)^{\frac{1}{2}} d t \\
& \leq \sqrt{t_{0}}\left(\int_{0}^{t_{0}} \int_{B_{1}}\left|w_{t}\right|^{2} d x d t\right)^{\frac{1}{2}} .
\end{aligned}
$$

Therefore,

$$
\begin{aligned}
& \int_{0}^{T^{\prime}} \int_{B_{1}}\left|w-w_{0}\right|^{2} t^{-\frac{3}{2}} d x d t \leq 2 \sqrt{T^{\prime}} \int_{0}^{T^{\prime}} \int_{B_{1}}\left|w_{t}\right|^{2} d x d t<+\infty \\
& \lim _{t \longrightarrow 0} t^{-\frac{1}{2}} \int_{\{t\} \times B_{1}}\left|w-w_{0}\right|^{2} d x=0 .
\end{aligned}
$$

Next, (1.5) gives,

$$
\begin{aligned}
& \int_{0}^{T^{\prime}} \int_{B_{1}}\langle\nabla u, \nabla w\rangle t^{-\frac{1}{2}} d x d t \\
= & \int_{0}^{T^{\prime}} \int_{B_{1}}\left\langle\nabla u, \nabla w-\nabla w_{0}\right\rangle t^{-\frac{1}{2}} d x d t+\int_{0}^{T^{\prime}} \int_{B_{1}}\left\langle\nabla u, \nabla w_{0}\right\rangle t^{-\frac{1}{2}} d x d t \\
= & \int_{0}^{T^{\prime}} \int_{B_{1}}\left\langle-u_{t}, w-w_{0}\right\rangle t^{-\frac{1}{2}} d x d t+\int_{0}^{T^{\prime}} \int_{B_{1}}\left\langle\nabla u, \nabla w_{0}\right\rangle t^{-\frac{1}{2}} d x d t \\
& -\int_{0}^{T^{\prime}} \int_{B_{1}}\left\langle A_{u}(\nabla u, \nabla u), w-w_{0}\right\rangle t^{-\frac{1}{2}} d x d t \\
\leq & \int_{0}^{T^{\prime}} \int_{B_{1}}\left\langle-u_{t}, w-w_{0}\right\rangle t^{-\frac{1}{2}} d x d t+2 \sqrt{2 T^{\prime} E\left(u_{0}\right)}\left(\int_{B_{1}}\left|\nabla w_{0}\right|^{2}\right)^{\frac{1}{2}} \\
& -\int_{0}^{T^{\prime}} \int_{B_{1}}\left\langle A_{u}(\nabla u, \nabla u), w-w_{0}\right\rangle t^{-\frac{1}{2}} d x d t .
\end{aligned}
$$

We will bound the third term from above. In the following calculation, energy nonincreasing condition and (3.9) guarantee that each quantity below is finite. Since $A_{u}(\nabla u, \nabla u)$ is perpendicular to $M$ at $u$ and $w=u-v$, we can apply Lemma 2.1 to the $L^{2}$ inner product of $A_{u}(\nabla u, \nabla u)$ and $w$ on $B_{1}$. Note that $w-w_{0}=$ $\left(u-u_{0}\right)-\left(v-v_{0}\right) \in H_{0}^{1}\left(B_{1}\right)$ for a.e. $t$ fixed. Thus, we can apply the interior gradient estimate (3.4) and Lemma 3.1 to the $L^{2}$ inner product of $|\nabla u|^{2}$ and $\left|w-w_{0}\right|^{2}$ on $B_{1}$. Hence,

$$
\begin{aligned}
& -\int_{0}^{T^{\prime}} \int_{B_{1}}\left\langle A_{u}(\nabla u, \nabla u), w-w_{0}\right\rangle t^{-\frac{1}{2}} d x d t \\
\leq & \lambda_{1} \int_{0}^{T^{\prime}} \int_{B_{1}}|\nabla u|^{2}|w|^{2} t^{-\frac{1}{2}} d x d t+\lambda_{2} \int_{0}^{T^{\prime}} \int_{B_{1}}|\nabla u|^{2}\left|w_{0}\right| t^{-\frac{1}{2}} d x d t \\
\leq & \lambda_{1} \int_{0}^{T^{\prime}} \int_{B_{1}}|\nabla u|^{2}\left|w-w_{0}\right|^{2} t^{-\frac{1}{2}} d x d t+\lambda_{2} \int_{0}^{T^{\prime}} \int_{B_{1}}|\nabla u|^{2}\left(\left|w_{0}\right|^{2}+\left|w_{0}\right|\right) t^{-\frac{1}{2}} d x d t
\end{aligned}
$$




$$
\begin{aligned}
\leq & \lambda_{1} C_{1} \epsilon_{2} \int_{0}^{T^{\prime}} \int_{B_{1}}\left|w-w_{0}\right|^{2} t^{-\frac{3}{2}} d x d t+4 \lambda_{1} C_{1} \epsilon_{2} \int_{0}^{T^{\prime}} \int_{B_{1}}\left|\nabla w-\nabla w_{0}\right|^{2} t^{-\frac{1}{2}} d x d t \\
& +\lambda_{2} \int_{0}^{T^{\prime}} \int_{B_{1}}|\nabla u|^{2}\left(\left|w_{0}\right|^{2}+\left|w_{0}\right|\right) t^{-\frac{1}{2}} d x d t,
\end{aligned}
$$

where $\lambda_{1}=C \sup _{M}|A|$ and $\lambda_{2}>0$ (changing from line to line in the computation above) depends only on $M$. The previous two inequalities and $32 \lambda_{1} C_{1} \epsilon_{2}<1$ give

$$
\begin{aligned}
& \int_{0}^{T^{\prime}} \int_{B_{1}}\langle\nabla u, \nabla w\rangle t^{-\frac{1}{2}} d x d t \\
\leq & \int_{0}^{T^{\prime}} \int_{B_{1}}\left\langle-u_{t}, w-w_{0}\right\rangle t^{-\frac{1}{2}} d x d t+\frac{1}{32} \int_{0}^{T^{\prime}} \int_{B_{1}}\left|w-w_{0}\right|^{2} t^{-\frac{3}{2}} d x d t \\
& +\frac{1}{4} \int_{0}^{T^{\prime}} \int_{B_{1}}|\nabla w|^{2} t^{-\frac{1}{2}} d x d t+N_{1},
\end{aligned}
$$

where

$$
\begin{aligned}
N_{1}= & \lambda_{2} \int_{0}^{T^{\prime}} \int_{B_{1}}\left(|\nabla u|^{2}+|\nabla v|^{2}\right)\left(\left|w_{0}\right|^{2}+\left|w_{0}\right|\right) t^{-\frac{1}{2}} d x d t \\
& +2 \sqrt{2 T^{\prime}\left(E\left(u_{0}\right)+E\left(v_{0}\right)\right)}\left(\int_{B_{1}}\left|\nabla w_{0}\right|^{2}\right)^{\frac{1}{2}}+\frac{\sqrt{T^{\prime}}}{2} \int_{B_{1}}\left|\nabla w_{0}\right|^{2} d x .
\end{aligned}
$$

Similarly,

$$
\begin{aligned}
& -\int_{0}^{T^{\prime}} \int_{B_{1}}\langle\nabla v, \nabla w\rangle t^{-\frac{1}{2}} d x d t \\
\leq & \int_{0}^{T^{\prime}} \int_{B_{1}}\left\langle v_{t}, w-w_{0}\right\rangle t^{-\frac{1}{2}} d x d t+\frac{1}{32} \int_{0}^{T^{\prime}} \int_{B_{1}}\left|w-w_{0}\right|^{2} t^{-\frac{3}{2}} d x d t \\
& +\frac{1}{4} \int_{0}^{T^{\prime}} \int_{B_{1}}|\nabla w|^{2} t^{-\frac{1}{2}} d x d t+N_{1} .
\end{aligned}
$$

Thus, combining (3.11) and (3.12), we get

$$
\begin{aligned}
& \int_{0}^{T^{\prime}} \int_{B_{1}}|\nabla w|^{2} t^{-\frac{1}{2}} d x d t \\
\leq & -\int_{0}^{T^{\prime}} \int_{B_{1}}\left\langle w_{t}, w-w_{0}\right\rangle t^{-\frac{1}{2}} d x d t+\frac{1}{16} \int_{0}^{T^{\prime}} \int_{B_{1}}\left|w-w_{0}\right|^{2} t^{-\frac{3}{2}} d x d t \\
& +\frac{1}{2} \int_{0}^{T^{\prime}} \int_{B_{1}}|\nabla w|^{2} t^{-\frac{1}{2}} d x d t+2 N_{1} \\
\leq & -\frac{1}{2 \sqrt{T^{\prime}}} \int_{\left\{T^{\prime}\right\} \times B_{1}}\left|w-w_{0}\right|^{2} d x+\frac{1}{2} \int_{0}^{T^{\prime}} \int_{B_{1}}|\nabla w|^{2} t^{-\frac{1}{2}} d x d t+2 N_{1},
\end{aligned}
$$


where the last inequality follows from integration by parts and (3.10). Therefore,

$$
\begin{aligned}
& \int_{0}^{T^{\prime}} \int_{B_{1}}|\nabla w|^{2} t^{-\frac{1}{2}} d x d t+\frac{1}{2 \sqrt{T^{\prime}}} \int_{\left\{T^{\prime}\right\} \times B_{1}}|w|^{2} d x \\
\leq & \frac{1}{\sqrt{T^{\prime}}} \int_{B_{1}}\left|w_{0}\right|^{2} d x+4 N_{1},
\end{aligned}
$$

and $C_{2}=\lambda_{2}$ in the lemma.

Now, under the condition of Theorem 1.1 that is, $u_{0}=v_{0}$, we have $N \equiv 0$ and thus $u(t, \cdot)=v(t, \cdot)$ in $L^{2}\left(B_{1}\right)$ for each $t \in\left[0, T^{\prime}\right]$. Therefore, it follows from an open and closed argument that $u=v$ a.e. on $[0, T] \times B_{1}$.

\section{Proof of Theorem 1.3}

Let $u$ be the weak solution of (1.2) in $\bigcap_{T>0} H^{1}\left([0, T] \times B_{1}, M\right)$ with initial data $u_{0} \in H^{1}\left(B_{1}, M\right)$ and boundary data $\gamma=\left.u_{0}\right|_{\partial B_{1}}$. First, define $E=\sup _{t \geq 0} E(u(t, \cdot))$. If $E<\epsilon_{1}$, then, by a similar argument used to obtain (3.4), we get

$$
|\nabla u|^{2}\left(t_{0}, x_{0}\right) \leq C_{1} E \max \left\{t_{0}^{-1},\left(1-\left|x_{0}\right|\right)^{-2}\right\} \leq C_{1} E\left[t_{0}^{-1}+\left(1-\left|x_{0}\right|\right)^{-2}\right],
$$

for $t_{0}>0$ and $x_{0} \in B_{1}$.

Second, we derive two estimates of the kinetic energy: one holds for a.e. $t_{0}>0$; the other holds for $t_{0}$ large enough.

Lemma 4.1. There exists $\epsilon_{3} \in\left(0, \epsilon_{1}\right)$, depending only on $M$, so that if $E \leq \epsilon_{3}$, then for a.e. $t_{0}>0$,

$$
\int_{\left\{t_{0}\right\} \times B_{1}}\left|u_{t}\right|^{2} d x \leq \frac{4}{t_{0}} \int_{0}^{t_{0}} \int_{B_{1}}\left|u_{t}\right|^{2} d x d t
$$

and there exist $T_{1}>0, \alpha_{1}>0$ and $C_{3}>0$ such that for a.e. $t_{0} \geq T_{1}$,

$$
\int_{\left\{t_{0}\right\} \times B_{1}}\left|u_{t}\right|^{2} d x \leq C_{3} \exp \left[-\alpha_{1}\left(t-T_{1}\right)\right] .
$$

Proof. Assume that $E<\epsilon_{1}$. Let $0<h<h_{0} \ll 1$. We define the difference quotient $D^{h} u=(u(t+h, x)-u(t, x)) / h$. Note that

$$
D^{h} u(t, \cdot) \in H_{0}^{1} \cap L^{\infty}\left(B_{1}\right) \text { for a.e. } t \text { fixed. }
$$

Thus, by equation (2.1), we get

$$
\begin{aligned}
& \frac{d}{d t} \int_{\{t\} \times B_{1}}\left|D^{h} u\right|^{2} d x \\
= & -2 \int_{\{t\} \times B_{1}}\left|\nabla D^{h} u\right|^{2} d x+2 h^{-1} \int_{\{t\} \times B_{1}}\left\langle A_{u}(\nabla u, \nabla u), D^{h} u\right\rangle d x \\
& -2 h^{-1} \int_{\{t\} \times B_{1}}\left\langle A_{u(t+h, x)}(\nabla u, \nabla u), D^{h} u\right\rangle d x \\
\leq & -2 \int_{\{t\} \times B_{1}}\left|\nabla D^{h} u\right|^{2} d x+\lambda_{1} \int_{\{t\} \times B_{1}}\left|D^{h} u\right|^{2}\left(|\nabla u|^{2}(t, x)+|\nabla u|^{2}(t+h, x)\right) d x,
\end{aligned}
$$


where $\lambda_{1}=C \sup _{M}|A|$, and we use (1.3) and Lemma 2.1] in the last inequality. For $t>0$, by (4.1), (4.4), and Lemma 3.1, we have

$$
\begin{aligned}
& \int_{\{t\} \times B_{1}}\left|D^{h} u\right|^{2}|\nabla u|^{2} d x \\
\leq & C_{1} E \int_{\{t\} \times B_{1}}\left|D^{h} u\right|^{2}\left[t^{-1}+\left(1-|x|^{2}\right)^{-2}\right] d x \\
\leq & 4 C_{1} E \int_{\{t\} \times B_{1}}\left|\nabla D^{h} u\right|^{2} d x+C_{1} E t^{-1} \int_{\{t\} \times B_{1}}\left|D^{h} u\right|^{2} d x .
\end{aligned}
$$

If $8 C_{1} \lambda_{1} E \leq 1$, then

$$
\begin{aligned}
& \frac{d}{d t} \int_{\{t\} \times B_{1}}\left|D^{h} u\right|^{2} d x \\
\leq & -\int_{\{t\} \times B_{1}}\left|\nabla D^{h} u\right|^{2} d x+t^{-1} \int_{\{t\} \times B_{1}}\left|D^{h} u\right|^{2} d x \\
\leq & -\left(C_{s}^{-1}-t^{-1}\right) \cdot \int_{\{t\} \times B_{1}}\left|D^{h} u\right|^{2} d x,
\end{aligned}
$$

where we apply the Sobolev inequality to $D^{h} u(t, \cdot) \in H_{0}^{1}\left(B_{1}\right)$ in the first inequality and $C_{s}>0$ is the Sobolev constant of $B_{1}$.

Thus, integrating over $\left[t_{0} / 2, t_{0}\right]$, (4.5) gives

$$
\int_{\left\{t_{0}\right\} \times B_{1}}\left|D^{h} u\right|^{2} d x \leq \frac{4}{t_{0}} \int_{0}^{t_{0}} \int_{B_{1}}\left|D^{h} u\right|^{2} d x d t
$$

On the other hand, let $I=\left[2 C_{s}, 2 C_{s}+1\right]$, and it is obvious that

$$
\inf _{t \in I} \int_{\{t\} \times B_{1}}\left|D^{h} u\right|^{2} d x \leq \int_{I} \int_{B_{1}}\left|D^{h} u\right|^{2} d x d t
$$

Thus, for $t>T_{1}=2 C_{s}+2$, (4.5) also implies that

$$
\int_{\{t\} \times B_{1}}\left|D^{h} u\right|^{2} d x \leq \int_{I} \int_{B_{1}}\left|D^{h} u\right|^{2} d x d t \cdot \exp \left[-C_{s}^{-1}\left(t-T_{1}\right) / 2\right]
$$

Letting $h \longrightarrow 0$ and by (2.15), Lemma 4.1 follows with $\epsilon_{3}=\min \left\{\epsilon_{1}, C_{1}^{-1} \lambda_{1}^{-1} / 8\right\}$, $C_{3}=\int_{I} \int_{B_{1}}\left|u_{t}\right|^{2} d x d t$ and $\alpha_{1}=C_{s}^{-1} / 2$.

Finally, assume that $E<\epsilon_{3}$ and $32 C C_{1} E \sup _{M}|A|<1$. In the calculation below, we first apply Hölder's inequality to the $L^{2}$ inner product of $u_{t}$ and $u\left(t_{2}, \cdot\right)-u\left(t_{1}, \cdot\right)$ on $B_{1}$. Meanwhile, we use (1.3), Lemma 2.1 and Lemma 3.1 to bound the $L^{2}$ inner product of $A_{u\left(t_{2}, x\right)}(\nabla u, \nabla u)$ and $u\left(t_{2}, \cdot\right)-u\left(t_{1}, \cdot\right)$ on $B_{1}$. Next, we deduce the last inequality from Cauchy's inequality and the assumption on the upper bound of $E$. 
That is, for a.e. $T_{1}<t_{1}<t_{2}$,

$$
\begin{aligned}
& \int_{B_{1}}\left\langle\nabla u\left(t_{2}, x\right), \nabla u\left(t_{2}, x\right)-\nabla u\left(t_{1}, x\right)\right\rangle d x \\
= & \int_{B_{1}}\left\langle-u_{t}\left(t_{2}, x\right)-A_{u\left(t_{2}, x\right)}(\nabla u, \nabla u), u\left(t_{2}, x\right)-u\left(t_{1}, x\right)\right\rangle d x \\
\leq & \sqrt{C_{s}}\left(\int_{B_{1}}\left|u_{t}\left(t_{2}, x\right)\right|^{2} d x\right)^{\frac{1}{2}}\left(\int_{B_{1}}\left|\nabla u\left(t_{2}, x\right)-\nabla u\left(t_{1}, x\right)\right|^{2} d x\right)^{\frac{1}{2}} \\
& +4 C C_{1} E \sup _{M}|A| \cdot \int_{B_{1}}\left|\nabla u\left(t_{2}, x\right)-\nabla u\left(t_{1}, x\right)\right|^{2} d x \\
\leq & 2 C_{s} \int_{B_{1}}\left|u_{t}\left(t_{2}, x\right)\right|^{2} d x+\frac{1}{4} \int_{B_{1}}\left|\nabla u\left(t_{2}, x\right)-\nabla u\left(t_{1}, x\right)\right|^{2} d x .
\end{aligned}
$$

Similarly,

$$
\begin{aligned}
& \int_{B_{1}}\left\langle\nabla u\left(t_{1}, x\right), \nabla u\left(t_{1}, x\right)-\nabla u\left(t_{2}, x\right)\right\rangle d x \\
\leq & 2 C_{s} \int_{B_{1}}\left|u_{t}\left(t_{1}, x\right)\right|^{2} d x+\frac{1}{4} \int_{B_{1}}\left|\nabla u\left(t_{1}, x\right)-\nabla u\left(t_{2}, x\right)\right|^{2} d x .
\end{aligned}
$$

Summing the two inequalities above, we get

$$
\int_{B_{1}}\left|\nabla u\left(t_{1}, x\right)-\nabla u\left(t_{2}, x\right)\right|^{2} d x \leq 4 C_{s} \int_{B_{1}}\left(\left|u_{t}\left(t_{1}, x\right)\right|^{2}+\left|u_{t}\left(t_{2}, x\right)\right|^{2}\right) d x .
$$

Therefore, Theorem 1.3 follows immediately from Lemma 4.1 by choosing $\epsilon_{0}=$ $\min \left\{\epsilon_{3}, C^{-1} C_{1}^{-1} \inf _{M}|A|^{-1} / 32\right\}$.

\section{EXAMPLE OF HARMONIC MAP HEAT FLOW NOT IN $V^{T}$}

In this section, we construct the unique weak solution $u \in \bigcap_{T>0} H^{1}([0, T] \times$ $\left.B_{1}, M\right)$ of (1.2) starting with small energy initial data $u_{0} \in H^{1} \cap C^{0}\left(\bar{B}_{1}, M\right)$ and boundary data $\gamma=\left.u_{0}\right|_{\partial B_{1}}$. In general, the weak solution $u$ is not in $V^{T}$.

Proposition 5.1. There exists $\epsilon_{4}>0$, depending only on $M$, so that given $u_{0} \in H^{1} \cap C^{0}\left(\bar{B}_{1}, M\right)$ with $E\left(u_{0}\right)<\epsilon_{4}$, there exists a unique weak solution in $\bigcap_{T>0} H^{1}\left([0, T] \times B_{1}, M\right)$ of (1.2) whose energy is non-increasing. Moreover, for $0 \leq t_{1}<t_{2}$

$$
\frac{1}{7} \int_{B_{1}}\left|\nabla u\left(t_{2}, x\right)-\nabla u\left(t_{1}, x\right)\right|^{2} d x \leq \int_{B_{1}}\left|\nabla u\left(t_{1}, x\right)\right|^{2} d x-\int_{B_{1}}\left|\nabla u\left(t_{2}, x\right)\right|^{2} d x .
$$

Remark 5.2. Recently, Colding and Minicozzi showed that the $H^{1}$ distance between a harmonic map and an $H^{1}$ map with the same boundary value can be controlled by their gap in energy, assuming energy is small; see Theorem 3.1 in [3]. This is a key ingredient in the proof of finite time extinction of the Ricci flow. Combined with Theorem 1.3 and Remark 1.4, our estimate (5.1) can be viewed as a parabolic version of their theorem.

Proof. First, we may approximate $u_{0}$ by a sequence of maps $u_{m 0} \in C^{\infty}\left(\bar{B}_{1}, M\right)$ in $H^{1} \cap C^{0}$ topology 3 By Theorem 1.1 in [2, there exists $\delta_{1} \in\left(0, \epsilon_{2}\right)$, depending

\footnotetext{
${ }^{3}$ Let $v_{m 0} \in C^{\infty}\left(\bar{B}_{1}, \mathbb{R}^{N}\right)$ be the global approximations of $u_{0}$, constructed in Theorem 3 on page 252 of [5]. Then $u_{m 0}$ could be the nearest point projection (onto $M$ ) of $v_{m 0}$.
} 
only on $M$, so that if $E\left(u_{m 0}\right)<\delta_{1}$, then the weak solution $u_{m} \in \bigcap_{T>0} W_{p}^{1,2} \cap$

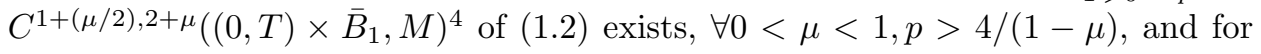
$0 \leq t_{1}<t_{2}$

$$
\int_{t_{1}}^{t_{2}} \int_{B_{1}}\left|\partial_{t} u_{m}\right|^{2} d x d t=E\left(u_{m}\left(t_{1}, \cdot\right)\right)-E\left(u_{m}\left(t_{2}, \cdot\right)\right)
$$

If $E\left(u_{0}\right)<\delta_{1} / 2$, then, by Lemma 3.2 and a diagonalization argument, there exists a subsequence (relabeled) of $u_{m}$ satisfying that for $\forall T>0, \partial_{t} u_{m} \rightarrow \partial_{t} u$ weakly in $L^{2}\left([0, T] \times B_{1}\right), u_{m} \longrightarrow u$ and $\nabla u_{m} \longrightarrow \nabla u$ strongly in $L^{2}\left([0, T] \times B_{1}\right)$. Note that the boundary data $\gamma_{m} \longrightarrow \gamma$ in $H^{1 / 2} \cap C^{0}\left(\partial B_{1}, M\right)$. Therefore, $u \in \bigcap_{T>0} H^{1}\left([0, T] \times B_{1}\right)$ is the weak solution of (1.2) with initial data $u_{0}$ and boundary data $\gamma=\left.u_{0}\right|_{\partial B_{1}}$. Moreover, $E(u(t, \cdot)) \leq E\left(u_{0}\right)$ for a.e. $t$ and there exists a zero measure set $I_{1} \subseteq(0, \infty)$ so that if $t_{1}, t_{2} \in I_{1}^{c}$ and $t_{1}<t_{2}$, then

$$
\int_{t_{1}}^{t_{2}} \int_{B_{1}}\left|u_{t}\right|^{2} d x d t \leq E\left(u\left(t_{1}, \cdot\right)\right)-E\left(u\left(t_{2}, \cdot\right)\right)
$$

Second, we will show that inequality (5.3) is actually equality for a.e. $0<t_{1}<t_{2}$. Let $0<h<h_{0} \ll 1$. Define $D^{h} u(t, x)=(u(t+h, x)-u(t, x)) / h$. Thus, for $0<t_{1}<t_{2}$

$$
\begin{aligned}
& \int_{t_{1}}^{t_{2}} \int_{B_{1}}\left|u_{t}\right|^{2} d x d t=\lim _{h \longrightarrow 0} \int_{t_{1}}^{t_{2}} \int_{B_{1}}\left\langle u_{t}, D^{h} u\right\rangle d x d t \\
= & \lim _{h \longrightarrow 0} \int_{t_{1}}^{t_{2}} \int_{B_{1}}-\left\langle\nabla u, \nabla D^{h} u\right\rangle d x d t-\int_{t_{1}}^{t_{2}} \int_{B_{1}}\left\langle A_{u}(\nabla u, \nabla u), D^{h} u\right\rangle d x d t .
\end{aligned}
$$

We will bound the second term. In the following calculation, we use (1.3) and Lemma 2.1] in the first inequality, apply the gradient estimate (4.1) in the second inequality, and use (4.4) and Lemma 3.1] in the last inequality. Thus,

$$
\begin{aligned}
& \left|\int_{t_{1}}^{t_{2}} \int_{B_{1}}\left\langle A_{u}(\nabla u, \nabla u), D^{h} u\right\rangle d x d t\right| \\
\leq & h C \sup _{M}|A| \int_{t_{1}}^{t_{2}} \int_{B_{1}}|\nabla u|^{2}\left|D^{h} u\right|^{2} d x d t \\
\leq & h C C_{1} E\left(u_{0}\right) \sup _{M}|A| \int_{t_{1}}^{t_{2}} \int_{B_{1}}\left[t^{-1}+(1-|x|)^{-2}\right]\left|D^{h} u\right|^{2} d x d t \\
\leq & t_{1}^{-1} h C C_{1} E\left(u_{0}\right) \sup _{M}|A| \int_{t_{1}}^{t_{2}} \int_{B_{1}}\left|D^{h} u\right|^{2} d x d t \\
& +4 h C C_{1} E\left(u_{0}\right) \sup _{M}|A| \int_{t_{1}}^{t_{2}} \int_{B_{1}}\left|\nabla D^{h} u\right|^{2} d x d t .
\end{aligned}
$$

\footnotetext{
${ }^{4} w \in W_{p}^{1,2}\left((0, T) \times \bar{B}_{1}, M\right)$ means that $u,|\nabla u|,\left|\nabla^{2} u\right|$ and $\left|u_{t}\right|$ are in $L^{p}\left((0, T) \times \bar{B}_{1}\right)$, and $u \in M$ for a.e. $(t, x) \in(0, T) \times \bar{B}_{1}$.
} 
For the first term,

$$
\begin{aligned}
& \int_{t_{1}}^{t_{2}} \int_{B_{1}}-\left\langle\nabla u, \nabla D^{h} u\right\rangle d x d t \\
= & \frac{1}{2 h} \int_{t_{1}}^{t_{2}} \int_{B_{1}}\left(|\nabla u(t, x)|^{2}-|\nabla u(t+h, x)|^{2}\right) d x d t+\frac{h}{2} \int_{t_{1}}^{t_{2}} \int_{B_{1}}\left|\nabla D^{h} u\right|^{2} d x d t \\
= & \frac{1}{2 h} \int_{t_{1}}^{t_{1}+h} \int_{B_{1}}|\nabla u|^{2} d x d t-\frac{1}{2 h} \int_{t_{2}}^{t_{2}+h} \int_{B_{1}}|\nabla u|^{2} d x d t+\frac{h}{2} \int_{t_{1}}^{t_{2}} \int_{B_{1}}\left|\nabla D^{h} u\right|^{2} d x d t .
\end{aligned}
$$

If $E\left(u_{0}\right)<C^{-1} C_{1}^{-1} \inf _{M}|A|^{-1} / 8$, then

$$
\begin{aligned}
& \int_{t_{1}}^{t_{2}} \int_{B_{1}}\left|u_{t}\right|^{2} d x d t \\
\geq & \lim _{h \longrightarrow 0} \frac{1}{2 h} \int_{t_{1}}^{t_{1}+h} \int_{B_{1}}|\nabla u|^{2} d x d t \\
& -\frac{1}{2 h} \int_{t_{2}}^{t_{2}+h} \int_{B_{1}}|\nabla u|^{2} d x d t-\frac{h}{8 t_{1}} \int_{t_{1}}^{t_{2}} \int_{B_{1}}\left|D^{h} u\right|^{2} d x d t \\
\geq & \lim _{h \longrightarrow 0} \frac{1}{2 h} \int_{t_{1}}^{t_{1}+h} \int_{B_{1}}|\nabla u|^{2} d x d t-\frac{1}{2 h} \int_{t_{2}}^{t_{2}+h} \int_{B_{1}}|\nabla u|^{2} d x d t .
\end{aligned}
$$

Define $f(t)=\int_{B_{1}}|\nabla u(t, x)|^{2} d x$ and $F(t)=\int_{0}^{t} f(s) d s$. Since $f \in L^{1}([0, T])$ for $\forall T \in(0, \infty), F^{\prime}(t)=f(t)$ for a.e. $t \in[0, T]$. Hence, there exists a zero measure set $I_{2} \subseteq(0, \infty)$ so that if $t \in I_{2}^{c}$, then

$$
\lim _{h \longrightarrow 0} \frac{1}{h} \int_{t}^{t+h} \int_{B_{1}}|\nabla u|^{2} d x d t=\int_{B_{1}}|\nabla u(t, x)|^{2} d x
$$

Therefore, if $t_{1}, t_{2} \in I_{2}^{c}$ and $0<t_{1}<t_{2}$, then

$$
\int_{t_{1}}^{t_{2}} \int_{B_{1}}\left|u_{t}\right|^{2} d x d t \geq E\left(u\left(t_{1}, \cdot\right)\right)-E\left(u\left(t_{2}, \cdot\right)\right)
$$

Combining with inequality (5.3), we get

$$
\int_{t_{1}}^{t_{2}} \int_{B_{1}}\left|u_{t}\right|^{2} d x d t=E\left(u\left(t_{1}, \cdot\right)\right)-E\left(u\left(t_{2}, \cdot\right)\right)
$$

if $t_{1}, t_{2} \in\left(I_{1} \cup I_{2}\right)^{c}$ and $0<t_{1}<t_{2}$.

Third, assume that $E\left(u_{0}\right)<\min \left\{\epsilon_{3} / 2, \delta_{1} / 2, C^{-1} C_{1}^{-1} \inf _{M}|A|^{-1} / 32\right\}$. Since $u_{m 0} \longrightarrow u_{0}$ in $H^{1}\left(B_{1}, M\right)$ topology, we may also assume that $E\left(u_{m 0}\right) \leq 2 E\left(u_{0}\right)$. 
Thus, for $0 \leq t_{1}<t_{2}$, using (4.1) and Lemmas 2.1] 3.1 and 4.15 we estimate

$$
\begin{aligned}
& \int_{B_{1}}\left\langle\nabla u_{m}\left(t_{2}, x\right), \nabla u_{m}\left(t_{2}, x\right)-\nabla u_{m}\left(t_{1}, x\right)\right\rangle d x \\
= & \int_{B_{1}}\left\langle-\partial_{t} u_{m}\left(t_{2}, x\right)-A_{u_{m}\left(t_{2}, x\right)}\left(\nabla u_{m}, \nabla u_{m}\right), u_{m}\left(t_{2}, x\right)-u_{m}\left(t_{1}, x\right)\right\rangle d x \\
\leq & \left(\int_{B_{1}}\left|\partial_{t} u_{m}\left(t_{2}, x\right)\right|^{2} d x\right)^{\frac{1}{2}}\left(\int_{B_{1}}\left|u_{m}\left(t_{1}, x\right)-u_{m}\left(t_{2}, x\right)\right|^{2} d x\right)^{\frac{1}{2}} \\
& +C \sup _{M}|A| \int_{B_{1}}\left|\nabla u_{m}\left(t_{2}, x\right)\right|^{2}\left|u_{m}\left(t_{1}, x\right)-u_{m}\left(t_{2}, x\right)\right|^{2} d x \\
\leq & \sqrt{t_{2}-t_{1}} \cdot\left(\int_{B_{1}}\left|\partial_{t} u_{m}\left(t_{2}, x\right)\right|^{2}\right)^{\frac{1}{2}}\left(\int_{t_{1}}^{t_{2}} \int_{B_{1}}\left|\partial_{t} u_{m}\right|^{2} d x d t\right)^{\frac{1}{2}} \\
& +C C_{1} E\left(u_{m}\left(t_{1}, \cdot\right)\right) \sup _{M}|A| \int_{B_{1}}\left(t_{2}-t_{1}\right)^{-1}\left|u_{m}\left(t_{1}, x\right)-u_{m}\left(t_{2}, x\right)\right|^{2} d x \\
& +C C_{1} E\left(u_{m}\left(t_{1}, \cdot\right)\right) \sup _{M}|A| \int_{B_{1}} \frac{\left|u_{m}\left(t_{1}, x\right)-u_{m}\left(t_{2}, x\right)\right|^{2}}{\left(1-\sqrt{x_{1}^{2}+x_{2}^{2}}\right)^{2}} d x \\
\leq & \frac{33}{16} \int_{t_{1}}^{t_{2}} \int_{B_{1}}\left|\partial_{t} u_{m}\right|^{2} d x d t+\frac{1}{4} \int_{B_{1}}\left|\nabla u_{m}\left(t_{2}, x\right)-\nabla u_{m}\left(t_{1}, x\right)\right|^{2} d x,
\end{aligned}
$$

where we use the fact that the energy of $u_{m}$ is non-increasing in the last inequality. Thus,

$$
\begin{aligned}
& \int_{B_{1}}\left|\nabla u_{m}\left(t_{1}, x\right)\right|^{2} d x-\int_{B_{1}}\left|\nabla u_{m}\left(t_{2}, x\right)\right|^{2} d x \\
= & \int_{B_{1}}\left|\nabla u_{m}\left(t_{1}, x\right)-\nabla u_{m}\left(t_{2}, x\right)\right|^{2} d x \\
& +2 \int_{B_{1}}\left\langle\nabla u_{m}\left(t_{2}, x\right), \nabla u_{m}\left(t_{1}, x\right)-\nabla u_{m}\left(t_{2}, x\right)\right\rangle d x \\
\geq & \frac{1}{2} \int_{B_{1}}\left|\nabla u_{m}\left(t_{1}, x\right)-\nabla u_{m}\left(t_{2}, x\right)\right|^{2} d x-5 \int_{t_{1}}^{t_{2}} \int_{B_{1}}\left|\partial_{t} u_{m}\right|^{2} d x d t .
\end{aligned}
$$

Hence, it follows from (5.2) that

$$
\frac{1}{7} \int_{B_{1}}\left|\nabla u_{m}\left(t_{1}, x\right)-\nabla u_{m}\left(t_{2}, x\right)\right|^{2} d x \leq \int_{B_{1}}\left|\nabla u_{m}\left(t_{1}, x\right)\right|^{2} d x-\int_{B_{1}}\left|\nabla u_{m}\left(t_{2}, x\right)\right|^{2} d x .
$$

By Lemma [3.2, there exists a zero measure set $I_{3} \subseteq(0, \infty)$ so that if $t \in I_{3}^{c}$, then there exists a subsequence (relabeled) of $u_{m}$ such that $u_{m}(t, \cdot) \longrightarrow u(t, \cdot)$ in $H^{1}\left(B_{1}, M\right)$ topology. Hence, if $0<t_{1}<t_{2}$ and $t_{1}, t_{2} \in\left(I_{1} \cup I_{2} \cup I_{3}\right)^{c}$, then

$$
\begin{aligned}
& \frac{1}{7} \int_{B_{1}}\left|\nabla u\left(t_{2}, x\right)-\nabla u\left(t_{1}, x\right)\right|^{2} d x \leq \int_{B_{1}}\left|\nabla u\left(t_{1}, x\right)\right|^{2} d x-\int_{B_{1}}\left|\nabla u\left(t_{2}, x\right)\right|^{2} d x \\
& \frac{1}{2} \int_{B_{1}}\left|\nabla u\left(t_{1}, x\right)\right|^{2} d x-\frac{1}{2} \int_{B_{1}}\left|\nabla u\left(t_{2}, x\right)\right|^{2} d x=\int_{t_{1}}^{t_{2}} \int_{B_{1}}\left|u_{t}\right|^{2} d x d t
\end{aligned}
$$

${ }^{5}$ Note that Lemma 4.1 holds for every $t_{0}>0$ for $u_{m}$, since $u_{m} \in C^{1+(\mu / 2), 2+\mu}\left((0, \infty) \times \bar{B}_{1}, M\right)$ and $\left\|u_{t}(t, \cdot)\right\|_{L^{2}\left(B_{1}\right)}$ is continuous in $t$. 
We can modify the definition of $u$ on $\left(I_{1} \cup I_{2} \cup I_{3}\right) \times B_{1}$ by taking limits. Therefore, the modified map solves (1.2) satisfying that the energy is non-increasing and the uniqueness for weak solutions of the harmonic map heat flow follows from Theorem 1.1 .

\section{Appendix A}

Proposition A.1. There exists $\epsilon_{5}>0$, depending on $M$, so that if $|x-y|<\epsilon_{5}$, then $\operatorname{dist}_{M}(x, y)<2|x-y|$, where $\operatorname{dist}_{M}(x, y)$ is the intrinsic distance between $x$ and $y$ on $M$.

Proof. If not, then there exists a sequence of $\left(x_{j}, y_{j}\right) \in M \times M$ such that $\left|x_{j}-y_{j}\right| \longrightarrow$ 0 but $\operatorname{dist}_{M}\left(x_{j}, y_{j}\right) \geq 2\left|x_{j}-y_{j}\right|$. Since $M$ is compact, there exist $x_{0} \in M$ and a subsequence (relabeled) of $\left(x_{j}, y_{j}\right)$ satisfying that $x_{j} \longrightarrow x_{0}$ and $y_{j} \longrightarrow x_{0}$. There exists $0<\delta_{1}<\sup _{M}|A| / 4$ such that the geodesic ball $B_{\delta_{1}}^{M}\left(x_{0}\right)$ centered at $x_{0}$ with radius $\delta_{1}$ is strictly geodesically convex. If $j$ is sufficiently large, then $x_{j}$ and $y_{j}$ are in $B_{\delta_{1}}^{M}\left(x_{0}\right)$. Let $l_{j}$ be the geodesic distance between $x_{j}$ and $y_{j}$, and $\gamma_{j}:\left[0, l_{j}\right] \longrightarrow B_{\delta_{1}}^{M}\left(x_{0}\right)$ be the unit speed minimizing geodesic joining $x_{j}$ and $y_{j}$. Thus,

$$
\begin{aligned}
\left|y_{j}-x_{j}\right|^{2} & =\int_{0}^{l_{j}} 2\left\langle\gamma_{j}(s)-x_{j}, \gamma_{j}^{\prime}(s)\right\rangle d s \\
& =\int_{0}^{l_{j}} \int_{0}^{s}\left(2\left|\gamma_{j}^{\prime}(\tau)\right|^{2}+2\left\langle\gamma_{j}(\tau)-x_{j}, \gamma^{\prime \prime}(\tau)\right\rangle\right) d \tau d s \\
& \geq \int_{0}^{l_{j}} \int_{0}^{s} 2\left(1-2 \delta_{1} \sup _{M}|A|\right) d \tau d s \\
& \geq \frac{l_{j}^{2}}{2}
\end{aligned}
$$

Therefore, $\operatorname{dist}_{M}\left(x_{j}, y_{j}\right) \leq \sqrt{2}\left|x_{j}-y_{j}\right|$, and this is a contradiction.

Proof of Lemma 2.1. If $|x-y| \geq \epsilon_{5}$, then $\left|(x-y)^{\perp}\right| /|x-y|^{2} \leq \epsilon_{5}^{-1}$. Otherwise, let $\gamma:[0, l] \longrightarrow M$ be the minimizing geodesic joining $y$ to $x$ with length $l \leq 2|x-y|$. Then

$$
\begin{aligned}
\left|(x-y)^{\perp}\right| & =\int_{0}^{l}\left\langle\gamma^{\prime}(s), V\right\rangle d s=\int_{0}^{l} \int_{0}^{s}\left\langle\gamma^{\prime \prime}(\tau), V\right\rangle d \tau d s \\
& \leq \sup _{M}|A| \cdot \frac{l^{2}}{2} \leq 2 \sup _{M}|A| \cdot|x-y|^{2},
\end{aligned}
$$

where $V=(x-y)^{\perp} /\left|(x-y)^{\perp}\right|$. Therefore, Lemma 2.1 follows immediately with $C=\max \left\{\epsilon_{5}^{-1}, 2 \sup _{M}|A|\right\}$.

\section{ACKNOWLEDGEMENTS}

The author would like to thank Professor Tobias Colding for suggesting this question and his continuous guidance. Also, the author is grateful to Jacob Bernstein and the anonymous referees for their useful comments. 


\section{REFERENCES}

[1] M. Bertsch, R. Dal Passo, and R. van der Hout, Nonuniqueness for the heat flow of harmonic maps on the disk, Arch. Ration. Mech. Anal. 161 (2002), no. 2, 93-112. MR.1870959 (2003a:35093)

[2] K.C. Chang, Heat flow and boundary value problem for harmonic maps, Ann. Inst. H. Poincaré Anal. Non Linéaire 6 (1989), no. 5, 363-395. MR1030856 (90i:58037)

[3] T.H. Colding and W.P. Minicozzi II, Width and finite extinction time of Ricci flow, Geom. Topol. 12 (2008), no. 5, 2537-2586. MR2460871 (2009k:53166)

[4] T.H. Colding and W.P. Minicozzi II, Width and mean curvature flow, Geom. Topol. 12 (2008), no. 5, 2517-2535. MR2460870 (2009k:53165)

[5] L.C. Evans, Partial Differential Equations, Graduate Studies in Mathematics 19, American Mathematical Society, Providence, RI, 1998 (reprinted with corrections 2002). MR.1625845 (99e:35001)

[6] A. Freire, Uniqueness for the harmonic map flow from surfaces to general targets, Comment. Math. Helv. 70 (1995), no. 2, 310-338. MR1324632 (96f:58045)

[7] A. Freire, Uniqueness for the harmonic map flow in two dimensions, Calc. Var. Partial Differential Equations 3 (1995), no. 1, 95-105. MR1384838 (97d:58058)

[8] A. Freire, Correction to: "Uniqueness for the harmonic map flow from surfaces to general targets" [Comment. Math. Helv. 70 (1995), no. 2, 310-338; MK1324632 (96f:58045)], Comment. Math. Helv. 71 (1996), no. 2, 330-337. MR.1396678 (97c:58032)

[9] D. Gilbarg and N. Trudinger, Elliptic partial differential equations of second order, reprint of the 1998 edition, Classics in Mathematics, Springer-Verlag, Berlin, 2001. MR.1814364 (2001k:35004)

[10] R.S. Hamilton, Harmonic maps of manifolds with boundary, Lecture Notes in Mathematics 471, Springer-Verlag, Berlin-New York, 1975. MR0482822(58:2872)

[11] F. Hélein, Harmonic maps, conservation laws and moving frames, second edition, Cambridge Tracts in Mathematics 150, Cambridge University Press, Cambridge, 2002. Translated from the 1996 French original. With a foreword by James Eells. MR.1913803 (2003g:58024)

[12] G. Lieberman, Second order parabolic differential equations, revised version 2005, World Scientific Publishing Co., Inc., River Edge, NJ, 1996. MR.1465184 (98k:35003)

[13] J. Nečas, Sur une méthode pour résoudre les équations aux dérivées partielles du type elliptique, voisine de la variationnelle, Ann. Scuola Norm. Sup. Pisa (3) 16 (1962), 305-326. French. MR0163054 (29:357)

[14] T. Rivière, Flot des applications harmoniques en dimension deux, preprint ENS-Cachan (1993). In "Applications harmoniques entre varietes": These de l'Universite Paris 6.

[15] M. Struwe, On the evolution of harmonic mappings of Riemannian surfaces, Comment. Math. Helv. 60 (1985), no. 4, 558-581. MR826871 (87e:58056)

[16] M. Struwe, Variational methods. Applications to nonlinear partial differential equations and Hamiltonian systems, second edition. Ergebnisse der Mathematik und ihrer Grenzgebiete (3) [Results in Mathematics and Related Areas (3)], 34. Springer-Verlag, Berlin, 1996. MR:1411681 (98f:49002)

[17] L. Wang, Remark on weak solutions, preprint. Available at http://math.mit.edu/ luwang/.

Department of Mathematics, Massachusetts Institute of Technology, 77 MassachuSetts Avenue, Cambridge, Massachusetts 02139

E-mail address: luwang@math.mit.edu

Current address: Department of Mathematics, Johns Hopkins University, 3400 N. Charles Street, Baltimore, Maryland 21218

E-mail address: 1wang@math.jhu.edu 